\title{
Traduire Rimbaud en allemand
}

Translating Rimbaud into German

Hermann H. Wetzel

\section{(2) OpenEdition \\ Journals}

\section{Édition électronique}

URL : https://journals.openedition.org/rief/7273

DOI : 10.4000/rief.7273

ISSN : 2240-7456

\section{Éditeur}

Seminario di filologia francese

\section{Référence électronique}

Hermann H. Wetzel, «Traduire Rimbaud en allemand », Revue italienne d'études françaises [En ligne], 11 | 2021, mis en ligne le 15 novembre 2021, consulté le 18 novembre 2021. URL : http:// journals.openedition.org/rief/7273; DOI : https://doi.org/10.4000/rief.7273

Ce document a été généré automatiquement le 18 novembre 2021.

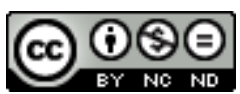

Les contenus de la RIEF sont mis à disposition selon les termes de la Licence Creative Commons Attribution - Pas d'Utilisation Commerciale - Pas de Modification 4.0 International. 


\title{
Traduire Rimbaud en allemand
}

\author{
Translating Rimbaud into German
}

Hermann H. Wetzel

1 Dans le présent article, je ne donnerai pas d'informations sur la réception allemande de Rimbaud en général ${ }^{1}$, ni de liste chronologique exhaustive des traductions. Je me concentrerai dans la première partie sur quelques difficultés de la traduction, en général et plus spécialement en allemand, et, dans la seconde, sur les chances qu'offrent des traductions raisonnées pour une connaissance approfondie de l'œuvre.

\section{Les difficultés de traduction}

2 Le langage poétique se caractérise par un usage spécifique de la langue, couramment considéré comme un écart par rapport à la norme. Il ne dévie pourtant pas seulement de la norme, mais élargit et approfondit les possibilités de la langue sous forme d'idiolecte. Or, il est difficile de reproduire dans la langue cible l'écart du texte à l'intérieur de la langue source tout en rendant compréhensible le texte dans la langue cible, parce que la traduction est toujours forcée de simplifier la polysémie du texte source. C'est pourquoi le plus souvent, la traduction est réellement, et pas seulement en apparence, plus pauvre et plus plate que le texte original.

3 Si l'on excepte les termes strictement techniques, les aires sémantiques des mots sont très différentes d'une langue à l'autre. Même si la dénotation est peut-être identique, les allusions et, à plus forte raison, les associations ne se recouvrent guère. À supposer qu'on soit d'accord sur ce dont il s'agit, la vieille "boîte " ${ }^{2}$ de Chant de guerre Parisien peut se traduire par "Schachtel», normalement en papier ou en carton, ou par " Büchse ", « Dose », en métal, ou par « Kasten », normalement en bois, avec des usages et, par conséquent, des associations très différentes. Ou pour prendre un exemple de la traduction de l'allemand en français: Jean Daive traduit le mot "VERBRACHT » au début du poème Engführung de Paul Celan avec «Dé/porté ». Sans entrer dans les détails ${ }^{3}$ on peut constater qu'il traduit correctement le terme technique. Or, Celan, lui, n'écrit pas « Deportiert », terme qui, en allemand même, est plus courant pour désigner 
la déportation vers les camps d'extermination, mais choisit un terme plutôt rare de la langue bureaucratique et juridique (qui, sous le Troisième Reich, évite les mots étrangers), pour marquer le procédé déshumanisant. En outre, le mot "verbracht » avec son préfixe destructif "ver- " est une paronomase d'un mot comme «Verbrechen" (crime) et de la dernière parole du Christ sur la croix: «(Es ist) vollbracht », « (Tout est) accompli ».

On voit bien que la traduction des allusions et associations touche très vite à ses limites et nécessite un commentaire. Le problème de l'hétérogénéité des sèmes d'une langue à l'autre redouble lors de la traduction des métaphores. Rimbaud étant connu pour ses métaphores vives ou, comme on les appelle en allemand, métaphores audacieuses ( kühne Metaphern»), leur traduction est une pierre de touche pour la qualité de la traduction. Ces métaphores foisonnent par exemple dans le Bateau ivre où elles appartiennent à des aires sémantiques très distantes, ce qui force le lecteur à chercher un tertium comparationis ou un sème commun qui souvent n'existe pas encore comme élément d'un mot lexicalisé. En défiant l'imagination du lecteur ces métaphores élargissent la manière de percevoir et de regarder le monde. À cette occasion, l'allemand profite de sa facilité à former des mots composés sans l'aide de prépositions, domaine dans lequel Celan ${ }^{4}$ est un des poètes modernes les plus experts.

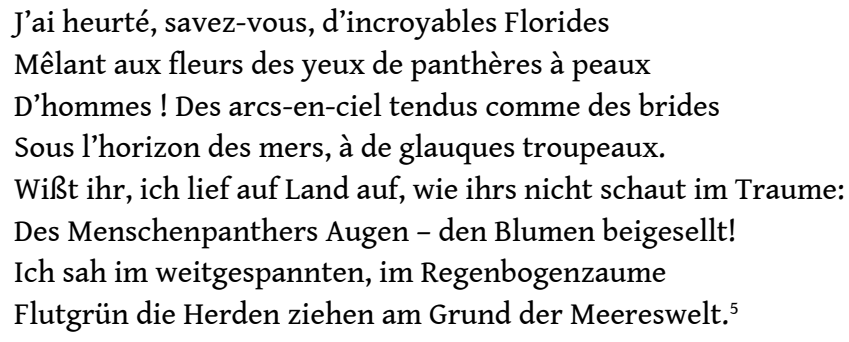

Les "yeux de panthères à peaux d'hommes ", littéralement "Augen der Panther in Menschenhaut ", est transformé par Celan en «Des Menschenpanthers Augen ». Avec " Menschenpanther ", « homme panthère », Celan exagère et exacerbe l'incompatibilité sémantique en confondant l'homme et la bête dans un seul mot. Il peut ainsi négliger le mot " peaux » qui rapproche l'homme de la bête, puisqu'à la différence de l'allemand, il peut aussi signifier fourrure ( Pelz»). De même, Celan raccourcit la comparaison " arcs-en-ciel [...] comme des brides» en une métaphore, sous la forme d'un mot composé insolite "Regenbogenzaum ». Le tertium comparationis, "tendus " précède le nouveau mot sous la forme d'un adjectif, «weitgespannt». La traduction de Celan montre aussi que cette facilité dans la formation des mots composés compense en quelque sorte les plus grandes difficultés de l'allemand à trouver des rimes: en surajoutant à «Meer(es)» («mers») le mot « welt» (« monde»), la rime est possible avec « beigesellt », littéralement associé, pour « mêlant ».

6 Homonymes et homophones peuvent être considérés comme des cas spéciaux de métaphores parce qu'ils rassemblent les deux termes de la métaphore dans un même mot appartenant à deux aires sémantiques différentes. Je présenterais plus bas avec Chant de guerre Parisien un exemple où Rimbaud déploie toute sa virtuosité sur ce terrain, ce qui met le traducteur devant des difficultés sémantiques très particulières.

7 Le problème de la traduction se renforce encore si l'on pense à l'engrenage du signifiant et du signifié au-delà de l'onomatopée. La cooccurrence entre son et contenu militaire dans le vers "Ils ont schako, sabre et tam-tam" du poème cité avec ses consonnes explosives et fricatives sonores, correspond presque parfaitement au vers 
allemand «Sie haben Tschako, Säbel und Tam-tam»; mais comment rendre le contraste avec la douceur phonique du vers suivant : « Non la vieille boîte à bougies »? J'y reviendrai dans les commentaires de la traduction de ce poème.

8 Du point de vue de la signification et de la traduction la rime est très proche de l'homophonie et de la paronomase. La ressemblance phonique suggère toujours un voisinage sémantique. Aussi la rime, au moins dans un poème bien construit, est-elle porteuse de signification. Une rime contrecarrée par une opposition sémantique a, volontairement ou involontairement, un effet critique ou comique ${ }^{6}$. Par conséquent, dans une traduction, on ne peut pas faire rimer n'importe quels mots ; rimer n'est pas automatiquement une qualité. Si l'on ne trouve pas une rime qui fasse sens ou soit au moins neutre, il vaut mieux ne pas rimer, pour éviter des effets fâcheux.

En outre, le vers français est fondamentalement différent du vers allemand. Celui-ci a un nombre fixe d'accents qui correspondent aux accents normaux des mots, alternant avec une ou plusieurs syllabes non accentuées, tandis que le vers français est caractérisé par un certain nombre de syllabes avec un accent obligatoire à la fin du vers et, selon la longueur du vers, à la césure. Les effets rythmiques se mesurent à l'écart par rapport au vers standard, sensible à l'oreille expert dès le début du poème. Si le poème français présente des effets rythmiques spéciaux, le traducteur cherchera à trouver un effet analogue en allemand. On pourra étudier un tel effet au premier vers du Chant de guerre Parisien traduit et analysé plus bas.

10 Voici, en quelques mots, le résumé de mes réflexions : pour ceux qui cherchent à pénétrer plus en profondeur l'usage proprement poétique de la langue chez Rimbaud la seule solution est d'apprendre le français et d'étudier les textes originaux. Mais pour leur faciliter la lecture et pour leur permettre de saisir l'originalité du texte en langue source, les traductions restent toujours un outil précieux. Je conseille l'utilisation des traductions dans d'autres langues même pour ceux qui savent parfaitement le français, voire même aux Français, parce qu'il y a des ambiguïtés sémantiques et des traits formels qui n'attirent notre attention que si l'on est confronté à la nécessité d'une traduction. Ainsi, les traductions forment un trésor d'interprétations supplémentaires dont on devrait profiter pour élucider la polyvalence des textes littéraires. La meilleure traduction, pourtant, peut être rendue encore plus transparente par un commentaire qui justifie ses choix entre plusieurs alternatives.

\section{Chances d'une édition bilingue avec traduction raisonnée : Chant de guerre Parisien}

11 La discussion des difficultés de traduction nous a menés plusieurs fois à conclure qu'une solution vraiment satisfaisante requiert obligatoirement un, voire deux commentaires qui se complètent réciproquement. C'est pour cette raison que je proposerai une sorte d'édition idéale ${ }^{7}$ qui présentera le texte en deux langues avec deux commentaires séparés: les informations éditoriales, lexicales, historiques, encyclopédiques seront complétées par la justification de la traduction proposée en allemand. Du coup, on évitera la faute majeure des traductions en général, et surtout des traductions de textes dits difficiles : la réduction de la complexité du texte source pour le rendre intelligible dans la langue cible. Le traducteur se trouve en face d'une difficulté double : faire sentir l'écart entre le texte original et le bon usage du temps de l'auteur dans la langue étrangère d'aujourd'hui. Sans commentaire, une expression 
française ambiguë se perd si elle vient à être nécessairement réduite à une seule signification en allemand. Le texte français n'étant pas forcé à un choix entre les deux significations, le commentateur peut tout simplement passer une ambiguïté sous silence.

La réduction forcée du texte par le traducteur est particulièrement gênante lorsqu'un auteur comme Rimbaud aime jouer sur les doubles sens et qu'un poème entier joue cette partition. Sans commentaire explicite, l'essentiel du poème se perdra dans la traduction en allemand ${ }^{8}$.

\section{CHANT DE GUERRE PARISIEN}

Le Printemps est évident, car

Du cœur des Propriétés vertes, Le vol de Thiers et de Picard

Tient ses splendeurs grandes ouvertes!

ô Mai ! quels délirants culs-nus !

Sèvres, Meudon, Bagneux, Asnières,

Écoutez donc les bienvenus

Semer les choses printanières !

Ils ont schako, sabre et tam-tam

Non la vieille boîte à bougies

Et des yoles qui n'ont jam, jam...

Fendent le lac aux eaux rougies!

Plus que jamais nous bambochons

Quand arrivent sur nos tanières

Crouler les jaunes cabochons

Dans des aubes particulières!

Thiers et Picard sont des Éros,

Des enleveurs d'héliotropes,

Au pétrole ils font des Corots :

Voici hannetonner leurs tropes...,

Ils sont familiers du Grand Truc !...

Et couché dans les glaïeuls, Favre

Fait son cillement aqueduc,

Et ses reniflements à poivre !

La Grand ville a le pavé chaud,

Malgré vos douches de pétrole,

Et décidément, il nous faut

Vous secouer dans votre rôle...

Et les Ruraux qui se prélassent

Dans de longs accroupissements,

Entendront des rameaux qui cassent

Parmi les rouges froissements !

\section{PARISER KRIEGSGESANG}

14 Frühling ist's, denn, ganz klar, Aus dem Herzen der Besitzungen, der grünen, 
Fliegen Thiers und Picard

Auf ihre offnen, glänzenden Bühnen!

Oh Mai! Welch irre Nackt-Schwänzchen!

Sèvres, Meudon, Bagneux, Asnières,

Hört die Willkommnen Frühlingskränzchen

Streuen, wie wenn es Saatgut wäre!

Sie haben Tschako, Säbel und tam-tam,

Doch keine alte Laterne sie schwenken.

Und Schiffchen, ganz ohne jam, jam ...

Durch des Sees gerötete Wasser sie lenken!

Mehr denn je wir feiern die Sause,

Wenn auf unsere armen Hütten

Die gelben Klunker wie 'ne Brause

In eigner Morgenröte sie schütten!

Thiers und Picard, sie spielen Eros,

Doch klaun sie nur Heliotropen;

In Petrol dafür sie malen Corots,

Da brummen schon ihre Tropen,

Sie sind vertraut mit dem Großen Trick!

Und Favre, in der Lilien Tiefen,

Lässt sprudeln seine Tränen quick

und schafft es mit Pfeffer, zu schniefen!

Der Hauptstadt Pflaster ist ganz heiß

Trotz euren öligen Duschen.

Bin ganz entschieden und ich weiß,

Dass ihr in eurer Rolle nicht dürft kuschen

Und die Junker machen sich's, die faulen,

bequem noch in langem Gesitze,

Bevor sie hören, wie die Äste aufjaulen

Unter dem roten Knistern der Blitze.

Sous le choc des événements historiques, de la guerre franco-prussienne de 1970/71, du siège de Paris et de la Commune, qui révolutionnent le système politique, mais aussi les idéologies et les choix esthétiques, Chant de guerre Parisien entame en fanfare la lettre de Rimbaud du 15 mai 1871 à Paul Demeny. C'est le premier de trois poèmes insérés qui figurent comme exemples de la " poésie nouvelle ", même si l'on a peut-être, à cause de leur ton polémique autant que de leur caractère de "circonstance ", des difficultés à les apprécier à leur juste valeur. Tous les trois sont des parodies : outre le Chant de guerre circassien de François Coppée qui se transforme en Chant de guerre Parisien, poésie à sujet historico-politique, un exemple de poésie sentimentale, Les Petites amoureuses de P. Glatigny, est réécrit sous le titre Mes petites amoureuses, et finalement Accroupissements parodie toute une catégorie de la poésie pieuse connue sous le nom d'« Élévations ». Ces poèmes-parodies se marient parfaitement au ton sarcastique et insolent de la lettre de ce garçon iconoclaste de seize ans qui glane ses idées sur l'art futur ça et là chez les présocialistes et des journalistes révolutionnaires ${ }^{10}$. Mais il ne se contente pas de démonter quelques-uns des poètes les plus renommés de son temps, il démontre en même temps les possibilités de leurs acquis poétiques au service d'une poésie moins autosuffisante et artistique vouée, elle, à un avenir rêvé. 


\section{Commentaire du texte français} guerre circassien, dans la collection Poèmes divers ${ }^{12}$, dont Rimbaud reprend aussi la forme. Mais même sans avoir connaissance de l'adjectif sous-jacent, on sentira le décalage entre un chant de guerre barbare et sa localisation dans la ville la plus civilisée du temps.

17 Les adjectifs finaux des deux titres riment et ne riment pas : ils sont très proches $d u$ point de vue phonique, mais, par contre, très distants du point de vue géographique et, surtout, politique. Une relation antagoniste analogue existe entre le traitement du sujet commun aux deux poèmes. Coppée glorifie le départ joyeux et hérö̈que, au printemps, des très lointains guerriers circassiens contre le tsar ${ }^{13}$, coloré de détails exotiques et richement rimé en huit quatrains. Les rimes riches à la manière des Parnassiens incitent Rimbaud à en faire au moins autant pour pouvoir ricaner en marge du poème : «Quelles rimes! ô ! quelles rimes! ». Ce renvoi est donc parodique en visant la poésie gratuite et pittoresque de Coppée et, en même temps, il est didactique en illustrant sous la forme d'un "psaume d'actualité » ${ }^{14}$ la tâche de la "littérature nouvelle » selon Rimbaud. En effet, le poème est d'une actualité brûlante (au sens propre!) car il précède immédiatement la Semaine Sanglante du 22 au 28 mai 1871, où Paris insurgé fut reconquis par le gouvernement des «Ruraux » réfugiés d'abord à Versailles, puis à Bordeaux, sous l'œil bienveillant des troupes prussiennes assiégeantes de Bismarck. La teneur dominante est claire et résumée avec évidence dans le titre : dans la guerre civile en cours, Rimbaud prend parti pour les révolutionnaires qui reprochent au gouvernement d'être lâche devant l'ennemi et de capituler par crainte d'une révolution sociale. Le Chant de guerre se nourrit de la conviction que la Commune triomphera militairement sur le gouvernement et sur Bismarck, même si les scènes du poème, comme elles sont présentées par la propagande du gouvernement, suggèrent presque tout au long du texte une idylle printanière.

Vers 1 : dès le début le ton est franchement ironique, il prétend que les événements réels contredisent ce qui est dit. Si le printemps, avec majuscule, était «évident » on n'aurait pas besoin de le dire. La constatation péremptoire va être justifiée par une proposition introduite par un "car », qui, faisant fi de toute règle métrique, sépare la dernière syllabe du vers pour créer un rythme bouffon : la conjonction qui introduit la raison alléguée est suivie d'une petite hésitation avant l'enjambement, ce qui enlève déjà toute sériosité à l'argument suivant, de même que la rime identique avec «Picard » qui ridiculise le ministre, rendu en quelque sorte responsable du début de la belle saison.

19 Vers 2 : le poème entier joue sur une double partition, dont l'idée se trouve déjà in nuce au début du poème de Coppée. Les mêmes mots font partie de deux systèmes sémantiques et scénarios contradictoires: sous le printemps la guerre. Si chez le Parnassien les deux aires sémantiques restent nettement séparées parce que les homonymes sont répétés dans leurs contextes respectifs, la guerre et le printemps, ils vont se confondre inséparablement chez Rimbaud, ce qui, on le verra, ne va pas sans quelque violence et grincement.

Coppée : Du Volga sur leurs bidets grêles, Les durs Baskirs vont arriver. 
Avril est la saison des grêles,

Et les balles vont le prouver.

Les neiges ont fini leurs fontes,

Les champs sont verts d'épis nouveaux ;

Mettons les pistolets aux fontes

Et les harnais d'or aux chevaux. ${ }^{15}$

Rimbaud soulignera au moyen de la confusion sémantique et iconographique au sein des mêmes mots (sans répétition dans un contexte différent) le caractère contre-nature et trompeur de cette mise en scène de la guerre civile par le gouvernement de Versailles.

21 Les prés verts, chez Coppée les «champs [...] verts », sont un élément obligatoire de l'iconographie printanière. Rimbaud remplace le syntagme "prés verts" par «Propriétés vertes ", avec un double profit: le mot «propriétés » renferme les lettres et les sons du mot " prés ", tous les deux désignant des terres, et remplace ainsi les prés sans perte ni de son, ni de contenu. En outre, il associe par-dessus le marché une signification économique et politique, en renvoyant au parti des propriétaires terriens, les « Ruraux $»^{16}$ qui soutiennent le gouvernement conservateur de Versailles contre les travailleurs de la Commune. Les « Propriétés vertes » (avec majuscule pour souligner la valeur du mot) rassemblent donc les deux isotopies, politique (guerre civile) et printanière, mais avec une nette prépondérance sur l'aspect politico-économique.

Vers 3-4: le vol des oiseaux est, à côté des prés verts, un autre élément obligatoire du printemps; l'homonyme "vol», avec sa deuxième signification, prendre ce qui appartient à autrui, s'insère aussi dans la sphère de la guerre civile: le chef du gouvernement, Thiers, fait voler des bombes et reconquiert Paris contre le gré des révolutionnaires. Qu'il s'agisse aussi d'un vol au sens de rapine et de violation est confirmé par l'apostrophe " enleveurs » au vers 18, et par la proximité de " Propriétés » au deuxième vers, ce qui rappelle la devise de Proudhon, très actuelle à l'époque, « La propriété, c'est le vol ».

parfaire le double sens du troisieme vers, les noms propres ne doivent pas seulement désigner les responsables politiques de la guerre civile mais, par surcroît, devraient se prêter à une déformation ornithologique pour s'insérer dans l'isotopie du printemps. Pour Rimbaud, le jeune prestidigitateur poétique, rien de plus facile! Au sein du personnel gouvernemental, il ne choisit pas les noms fortuitement. En premier lieu, bien sûr, il nomme Thiers, le premier responsable politique, mais après, pourquoi pas le général Vinoy, commandant de l'armée des assiégeants, ou un autre militaire, avant Picard, le ministre de l'intérieur? Tous les noms propres ne se prêtent pas au jeu : le couple «Thiers et Picard» renferme comme par hasard les syllabes nécessaires pour construire deux nouveaux noms d'oiseaux. [tjzRzepikaR] se compose de «tierce, fabriqué de tiercelet $»^{17}$, nom du mâle de certains oiseaux de proie plus petit d'un tiers que la femelle; et de pic, l'oiseau grimpeur bien connu. Par deux modifications dépréciatives apportées aux suffixes, l'un, diminutif, supprimé (-let), et l'autre, péjoratif, ajouté (-ard), les noms d'oiseaux se dévoilent comme des noms propres des adversaires politiques de la Commune révolutionnaire. Cette paire de noms politiques ou d'oiseaux enlaidis lui tient spécialement à cœur, comme le soulignera le vers 17 , où elle va être répétée.

Vers 5: un argument supplémentaire pour la lecture ornithologique de "Thiers et Picard » se trouve au début de la deuxième strophe : «culs-nus » n'est pas seulement 
une insulte, mais aussi un terme formé d'après des noms d'oiseaux tels que cul-blanc (pétrel), cul-rousset (fauvette), cul-rouge (pic épeiche), et confirme la classification des deux noms d'hommes politiques dans le registre du printemps. En même temps «culnu » est un synonyme de «Éros » avec majuscule, la personnification mythologique de l'amour, un petit Amour tout nu, élément fréquent de l'imagerie printanière, qui réapparaît au vers 17 avec les deux noms propres.

Vers 5-16: la deuxième, troisième et quatrième strophes déploient une fête populaire factice cachant en réalité, derrière la propagande des Versaillais, la guerre civile. Pour l'explication des détails, je renvoie aux commentaires de Steve Murphy et André Guyaux. Ce que je retiens sous l'angle de vue de la traduction, c'est que celle-ci a du mal à maintenir le double sens systématique, le traducteur devant le plus souvent choisir des termes qui appartiennent ou à l'isotopie du printemps ou à celle de la guerre.

Vers 10: l'euphonie du vers 10, qui s'accorde parfaitement avec l'idylle, contraste vivement avec le vers 9 , qui déploie une panoplie guerrière bruyante ou une parade de fête. La négation souligne qu'il ne s'agit pas, comme on pourrait croire, d'une illumination festive liée au vers 11 et à la citation de la joyeuse chanson enfantine Il était un petit navire, mais de l'imagerie de la guerre civile avec les eaux rougies par le sang ou le reflet des incendies ${ }^{18}$.

27 Vers 17: «des Éros » est une formule au goût du jeune Rimbaud parce qu'on peut la lire, avec un peu de liberté, de trois manières : d'abord correctement [dezeRos] avec la signification d'Amours, de putti, qui renvoient aux «culs-nus $»^{19} \mathrm{du}$ cinquième vers ; puis comme "des Héros » [dzeRo], des demi-dieux et finalement comme « des Zéros " [dzzeRo], des nuls. La première s'intègre au tableau du printemps, la troisième est encore une injure.

Vers 18 : les « héliotropes » ne sont pas identiques aux tournesols, fleurs automnales; Rimbaud connaît sa botanique. Au lieu de dérober le feu à Hélios comme le héros Prométhée, pour le donner avec toute la civilisation aux hommes, les dirigeants versaillais utilisent le feu pour la détruire.

Vers 20: les «tropes", c'est-à-dire les ornements des discours officiels qui bourdonnent comme des hannetons, se transforment en « troupes guerrières ".

Vers 21 : le «Grand Truc», que l'on peut confondre avec le «Grand Turc », évoque Bismarck.

31 Les vers 22-24 rappellent une scène souvent représentée dans les caricatures du temps où Favre, ridiculisé comme capitulard, semble avoir pleuré pendant les pourparlers engagés avec Bismarck en vue de l'armistice et de la paix, en s'aidant avec du poivre.

Les deux dernières strophes quittent le scénario de la guerre civile déguisée en fête pour passer directement à l'attaque. Au moment où Rimbaud écrit le poème, il croit encore à la victoire de la Commune, qu'il soutient avec ce chant de guerre mobilisateur. Les «accroupissements" du vers 30 rappellent le troisième poème de la lettre à Demeny qui porte justement ce titre.

\section{Commentaire de la traduction allemande}

En lisant le commentaire joint au texte français on aura tôt fait de se demander si la traduction d'un tel texte n'est pas impossible. Mais, chose curieuse, les traducteurs n'hésitent pas, soit qu'ils n'aient pas vu les difficultés, soit qu'ils y voient un défi. Je 
démarquerai ma traduction de deux autres : d'un côté, la plus solide, œuvre de Walther Küchler $^{20}$, qui, lui aussi, reproduit le texte original en face. Il n'a pas d'ambitions poétiques propres et se met au service du texte, il ne fait donc pas de «Nachdichtungen» (réécriture libre) comme Paul Zech" ${ }^{21}$, ni une traduction sophistiquée qui utilise toute sorte d'artifices typographiques, néologismes et motsvalises comme Hans Therre et Rainer G. Schmidt ${ }^{22}$ pour faire sentir même sans l'appui du texte français la complexité de l'original.

La remarque marginale ironique de Rimbaud sur ses propres rimes rend obligatoire une traduction rimée, parce que la rime raillée fait expressément partie du message poétologique de la lettre. Avec ses rimes, $\mathrm{K}$ garde le ton bouffon et dédaigneux, sans pourtant essayer d'imiter le jeu ambigu du double sens, ce qui affaiblit sensiblement le côté sarcastique et agressif de l'original, tandis que $\mathrm{Th} / \mathrm{Sch}$ renoncent à la rime et cherchent à remplacer l'ironie des rimes riches avec d'autres trouvailles.

Tous traduisent unanimement le titre par Kriegsgesang, ce qui dénote bien la fonction du "psaume ", l'exhortation à la résistance, même si l'on ne connaît pas le titre parodié. Th/Sch, pour mettre en évidence visuelle la relation du poème aux événements de la Commune de Paris ajoutent en bas de la page du poème, sans légende pourtant, une gravure montrant la destruction de la colonne Vendôme. Mais pas plus que $\mathrm{K}$ ils ne mentionnent le poème parodié de Coppée, ni le contexte de la lettre poétologique à Demeny qui manque d'ailleurs dans toutes les traductions sans commentaires. Conséquence fâcheuse : la dimension parodique et poétologique du poème se perd.

Vers 1 : le rythme trébuchant du vers français et la rime «car/Picard» doivent être conservés en allemand pour garder l'effet cocasse et ironique. K traduit : « Der Frühling kam, das ist sonnenklar ». On ne comprend pas pourquoi le traducteur change le temps du verbe. Le prétérit "kam» contredit l' «actualité» du "psaume». L'ironie de «évident », par contre, se manifeste dans l'hyperbole du « sonnenklar » (clair comme le soleil) qui rime avec «Picard». Th/Sch: «Der Lenz machts evident, denn». Le ton ironique est rendu par la forme raccourcie et familière de « machts » au lieu de « macht es », par le vocabulaire vieillot, «Lenz» au lieu de «Frühling », l'allitération finale («Lenz [ts] machts [ts] ») et le mot savant « evident » au lieu de «offensichtlich» ou «klar».

Verse 2-4: pour garder le double sens j'ai conservé aussi bien « Herz » pour « cœur » que « Besitzungen » pour « Propriétés ». "Cœur » et « Herz » ont tous les deux un côté sentimental, qui va très bien avec l'isotopie du printemps, et une signification métaphorique, qui prend un sens local en combinaison avec « Propriétés ». Le contraste entre les deux significations devient sensible si l'on regarde la nature explosive des dons des Ruraux qui pleuvent (volent) sur la ville. Le côté cordial se perd dans la traduction de Th/Sch «aus den Zentren » aussi bien que chez $\mathrm{K}$ " aus den grünen Gehegen ». Avec «Gehegen », enclos, se perd le renvoi au parti des propriétaires. Th/ Sch essaient de rattraper la majuscule insolite du mot « Propriétés » par des majuscules de l'adjectif ${ }^{23}$ de couleur "GRÜNEN », et sa signification politique par le remplacement de la désignation locale ("Propriétés ») par la mention des propriétaires ("Besitzer »).

L'homonymie de vol est presque ${ }^{24}$ impossible à traduire en allemand sans perte ; dans toutes les traductions allemandes, sauf une, le sème de prise illégale est perdu. $\mathrm{K}$ : "Man sieht aus den grünen Gehegen/ Den Flug von Thiers und Picard/ Weit offenen Glanzes sich regen// ». Personnellement j'ai opté avec le verbe «fliegen» pour 
l'isotopie du printemps pour souligner l'association avec les drôles d'oiseaux Thiers et Picard. Th/Sch, par contre, investissent beaucoup de sagacité et de créativité dans leur traduction: "startet die Bande von Thiers \& Picard/ zu ganz bombastischem HöhenfLUG! Avec «bombastisch» (pompier), ils gardent au moins une allusion aux splendeurs des bombes de pétrole (vers 15 «cabochons», vers 19 "pétrole») et un écho du vers 3 ( «bambochons»). L'esperluette \& renvoie au monde économique ${ }^{25} \mathrm{du}$ parti des propriétaires. Le double sens de «vol » est sauvé par la typographie étrange qui déforme le mot «Flug » (vol). Pour pouvoir profiter de l'attention attirée par les majuscules insolites en allemand, ils choisissent le mot composé "Höhenflug " (vol dans les hauteurs, envolée) qui permet d'écrire le mot « Flug ", à présent second terme d'un mot composé, avec une minuscule initiale et de mettre en relief les trois dernières lettres en capitales «-f LUG». Ainsi, ils peuvent faire ressortir le mot vieilli «Lug » (mensonge), et avec l'introduction du terme «Bande», groupe de brigands, pour les deux hommes politiques, la mise en scène mensongère de la guerre civile comme printemps devient évidente.

39 Vers 8: au lieu des très vagues « choses printanières " j'ai choisi "Frühlingskränzchen », petites couronnes printanières, pour gagner une rime cocasse avec la traduction ornithologique de "culs-nus », « Nackt-Schwänzchen », formé par analogie avec les noms d'oiseaux français en «cul-», d'après des noms allemands composés avec «-schwänzchen », comme " Rotschwänzchen » (rouge-queue).

Vers 15 : j'ai traduit "cabochons", pierres précieuses polies mais non taillées en facette, par «Klunker ${ }^{26}$, gros bijou faux; «Schnecken», escargots, de K ou " Kopf=Nüsse », petits coups contre la tête, de Th/Sch me paraissant trop loin du texte.

Vers 17-20: les différentes allusions du texte français doivent être confiées au commentaire pour ne garder dans la traduction allemande que la dominante de la guerre civile. Th/Sch s'évertuent à sauver le ton agressif en s'éloignant sensiblement du texte : "Thiers \& Picard ", " das sind die H=EROtomaten ». Les "H=EROtomaten » essaient de sauver Heros et Eros, combiné avec "automate », mais avec une fâcheuse association avec tomates.

Vers 30: je traduis "accroupissement» avec "Gesitze», pour rappeler les «Propriétés », « Besitzungen » du deuxième vers.

\section{NOTES}

1. Voir M. Gsteiger, Französische Symbolisten in der deutschen Literatur der Jahrhundertwende (1869-1914), Bern und München, Francke, 1971; H. H. Wetzel, « Réflexions lors d'une tentative de traduire Rimbaud en allemand", dans S. Sacchi, (dir.), Rimbaud, le poème en prose et la traduction poétique, Tübingen, Narr, 1988, p. 153-165 ; Id., "Paul Celan traducteur du Bateau ivre », dans Parade Sauvage, 8, 1991, p. 71-77 ; Id., «Rimbaud en Allemagne », dans Rimbaud Vivant, 30, 1991, p. 3-8 ; Id., "La réception de Rimbaud par les auteurs de l'ancienne RDA », dans Parade Sauvage, 15, 1998, p. 91-107.

2. Voir infra. 
3. Voir E. Dueck, L'Étranger intime. Les traductions françaises de l'œuvre de Paul Celan (1971-2010), Berlin, De Gruyter, 2014, p. 154 et sq.

4. Traduction de P. Celan, dans Id., Gesammelte Werke, t. IV, Übertragungen I (Zweisprachig), Frankfurt am Main, Suhrkamp, 1983, p. 103-111.

5. Ibid., p. 104-105

6. Voir infra la remarque marginale de Rimbaud à côté de Chant de guerre Parisien: "Quelles rimes ! ô quelles rimes ! » où il se moque de la richesse de ses propres rimes et de celles du poème parodié.

7. Projet présenté lors du Colloque de Ratisbonne (1995), mais jamais réalisé : T. Klinkert et H. H. Wetzel, (dir.), Traduction = Interprétation, Interprétation = Traduction. L'exemple Rimbaud, Actes du Colloque de Ratisbonne (21-23 septembre 1995), Paris, Champion, «Champion-Varia $16 », 1998$, p. 18-23.

8. Voir H. H. Wetzel, « Rimbauds Chant de guerre Parisien als Beispiel engagierter Dichtung », dans Germanisch-Romanische Monatsschrift, 27, 1977, p. 426-442.

9. A. Rimbaud, Euvres complètes, éd. A. Guyaux, Paris, Gallimard, «Bibliothèque de la Pléiade », 2009, p. 119-120.

10. Voir H. H. Wetzel, « Deux singularités », dans Parade sauvage, 3, 1986, p. 110-112.

11. Je m'appuie sur le commentaire de l'édition A. Rimbaud, CEuvres complètes, cit., p. 848-850 ; sur S. Murphy, "Chant de guerre Parisien: essai d'archéologie symbolique», dans Parade sauvage. Bulletin, 7, septembre 1991, et sur mes propres recherches.

12. F. Coppée, Chant de guerre circassien, in Id., Le Reliquaire, Paris, Lemerre, 1866, p. 127-129.

13. Les commentaires hésitent sur la guerre historique dont il s'agit. Mais peu importe: les peuples du Caucase sont connus pour leurs conflits permanents avec la Russie. Le détail mentionné du vieux chef qui bourre d'un geste dédaigneux son fusil avec un ukase du tsar, semble indiquer une sédition contre l'autorité russe. «Que le plus vieux chef du Caucase/ Bourre, en présence des aînés,/ Avec le vélin d'un ukase/ Les longs fusils damasquinés ! », Ibid., p. 128, v. 9-12.

14. La lettre commence ainsi : "J'ai résolu de vous donner une heure de littérature nouvelle ; je commence de suite par un psaume d'actualité », A. Rimbaud, «Lettre à Paul Demeny du 15 mai 1871 », dans Id., Euvres Complètes, cit., p. 342-349, p. 342.

15. F. Coppée, Chant de guerre circassien, dans Id., Le Reliquaire, cit., p. 127-128.

16. Voir vers 29 «Krautjunker», gentilhomme de chou, hobereau, dans la terminologie de Karl Marx.

17. Le Robert cite Régnier qui écrit « Tiercelets des poètes » au sens de poètes insignifiants.

18. S. Murphy (art. cit., p. 24) m'a d'ailleurs mal compris : sous l'influence d'une fête printanière illusoire la rime «bougie : rougies" pourrait suggérer de simples reflets de lanternes ou de lampions, qui n'existent expressément pas en réalité. Il n'y a ni lampions ni « Corots », seulement les reflets des incendies.

19. Une caricature visant Louis Philippe que j'ai déjà présentée dans Parade sauvage, 3, 1986, p. 110 comme source d'inspiration possible de Rimbaud, L'amour et l'idole des Français d'Auguste Bouquet dans La Caricature du 7 mars 1833, où l'on voit voltiger au centre un Amour bien gras vu de dos, en " cul-nu », et d'en haut avec une perruque, un gros nez et un carquois en forme de parapluie au milieu d'une guirlande de menottes, balances déséquilibrées, baïonnettes, pistolets et canons avec la légende « Voyez sa guirlande, voyez les fleurs qu'il répand sur la terre. Voyez! ». La confusion de Thiers avec la poire de Louis Philippe est fréquente dans la caricature aux temps de la Commune ; voir S. Murphy, art. cit., fig. IV, V, XV, XXXXVII.

20. La première édition de 1946 est le fruit du loisir forcé du professeur de philologie romane démis de son poste dès 1933. A. Rimbaud, Sämtliche Dichtungen. Französisch und Deutsch, éd. et tr. W. Küchler, Heidelberg, L. Schneider, « Weltliteratur », 1978, p. 84-85 (dorénavant K).

21. P. Zech, Sämtliche Dichtungen des A. Rimbaud. Nachdichtung [1944], München, dtv, 1963, p. 43. 
22. A. Rimbaud, Das trunkene Schiff. Gedichte, éd. et tr. H. Therre und R. G. Schmidt, München, Matthes \& Seitz, 1980 (dorénavant Th/Sch). Il y a, en outre, un très bref commentaire (Th/Sch : p. 290-291) qui parle de la Commune.

23. La majuscule initiale est de rigueur pour tous les noms dans la langue allemande.

24. Voir infra.

25. L'abréviation française « et Cie » s'écrit en allemand «\& Co».

26. La même traduction dans: A. Rimbaud, Seher-Briefe. Lettres du voyant, éd. et tr. W. von Koppenfels, Mainz, Dieterich, « Excerpta classica VII », 1990, p. 17.

\section{RÉSUMÉS}

Quelques remarques générales sur les difficultés et les chances de traduire Rimbaud en allemand sont à la base d'un projet de traduction raisonnée, c'est-à-dire d'une édition bilingue de Chant de guerre Parisien avec un commentaire expliquant le texte original et ses spécificités suivie d'une traduction en allemand avec un autre commentaire séparé qui discute et justifie les solutions choisies.

After a short review of the difficulties of translation from French to German language the article presents the project of an edition in two languages with two separated commentaries : the first explains the original text of Chant de guerre Parisien, the second discuss and justifies the choices of the translation.

\section{INDEX}

Mots-clés : Rimbaud (Arthur), Chant de guerre Parisien, traduction, commentaire, Allemagne Keywords : Rimbaud (Arthur), Chant de guerre Parisien, translation, commentary, Germany 